\title{
Validación de la Escala Competencia para el Cuidado en el Hogar en Adultos con Cáncer
}

\author{
Validation of the Competency Scale for Cancer Patient in-Home Care \\ Validação da Escala de Competências para Cuidados na Casa em \\ Adultos com Câncer
}

Revista Cuidarte

Rev Cuid. May - Ago 2021; 12(2): e1210

doi) http://dx.doi.org/10.15649/cuidarte.1210

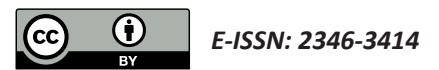

(1) Gloria Mabel Carrillo

(1) Elizabeth Vargas Rosero²

(1) Olga Janeth Gómez Ramírez ${ }^{3}$

1 Enfermera Magister en Enfermería. Doctora en Enfermería. Profesora Asociada Universidad Nacional de Colombia Facultad de Enfermería. Sede Bogotá. Autora de Correspondencia

E-mail: gmcarrillog@unal.edu.co

2 Enfermera. Magister en Enfermería. Doctora en Comunicación. Profesora Asociada Universidad Nacional de Colombia Facultad de Enfermería. Sede Bogotá.

E-mail: evargasr@unal.edu.co

3 Enfermera Magister en Enfermería. Doctora en Enfermería. Profesora Asociada Universidad Nacional de Colombia Facultad de Enfermería. Sede Bogotá.

E-mail: ojgomezr@unal.edu.co

\section{Resumen}

Introducción: Las competencias para el cuidado de adultos con cáncer en tratamiento son fundamentales para lograr adecuados procesos de transición hospital-hogar y comportamientos de automanejo en salud. Objetivo: validar la estructura factorial hipotética de la escala competencia para el cuidado en el hogar en adultos con diagnóstico de cáncer que se encuentran en tratamiento activo. Materiales y Métodos: estudio transversal de validación de instrumentos en el que participaron 250 adultos con diagnóstico de cáncer en tratamiento, se utilizó la técnica de análisis factorial confirmatorio utilizando el método de componentes principales y rotación varimax y el alfa de Cronbach para determinar la confiabilidad. Resultados: se obtiene una versión de la escala competencia para el cuidado en adultos con cáncer, con 17 items y 5 aspectos (factores): aprender, anticipar, autoregular, armonizar y apoyar. El alfa de Conbrach de la escala es de 0.764. Conclusiones: la escala competencia para el cuidado-5A retoma los planteamientos de la teoría de automanejo individual y familiar, evalúa de forma integral las habilidades requeridas en personas con cáncer para facilitar la transición hacia la autogestión del cuidado en el hogar.

Palabras clave: Neoplasias; Psicometría; Cuidados de Enfermería en el Hogar; Automanejo.
Recibido: abril 16 de 2020

Aceptado: febrero 2 de 2021

Publicado: mayo 18 de 2021 $\square *$ Correspondencia Gloria Mabel Carrillo

E-mail: gmcarrillog@unal.edu.co 


\title{
Validation of the Competency Scale for Cancer Patient in-Home Care
}

\begin{abstract}
Introduction: Caregiving competencies for cancer patients under treatment are fundamental to achieve an adequate hospital-home transition and health self-management behaviors. Objective: To validate the hypothetical factor structure of the competency scale for in-home care of cancer patients who are undergoing active cancer treatment. Methodology: A crosssectional study instrument validation was conducted with 250 cancer patients under treatment. The confirmatory factor analysis techniquewas used to determine reliability by using the principal component analysis method, varimax rotation and Cronbach's alpha. Results: A version of the Competency Scale for Cancer Patient In-Home Care was administered containing 17 items and 5 approaches: learning, anticipating, self-regulating, harmonizing, and supporting. Cronbach's alpha of the scale was 0.764. Conclusions: The 5-A Competency Scale for Cancer Patient InHome Care takes up the approaches of the individual and family self-management theory and comprehensively assesses the skills required by cancer patients to facilitate the transition to caregiving self-management at home.
\end{abstract}

Key words: Neoplasms; Psychometrics; Home Health Nursing; Self-Management

\section{Validação da Escala de Competências para Cuidados na Casa em Adultos com Câncer}

\section{Resumo}

Introdução: As competências para o cuidado de adultos com câncer em tratamento são fundamentais para o alcance de processos adequados de transição hospital-casa e comportamentos de autogestão em saúde. Objetivo: validar a estrutura fatorial hipotética da escala de competência para atenção domiciliar em adultos com diagnóstico de câncer que estão em tratamento ativo. Materiais e Métodos: estudo transversal de validação de instrumento do qual participaram 250 adultos com diagnóstico de câncer em tratamento, utilizando-se a técnica de análise fatorial confirmatória, utilizando o método de componentes principais e rotação varimax e o alfa de Cronbach para determinar a confiabilidade. Resultados: obteve-se uma versão da escala de competências para cuidar de adultos com câncer, com 17 itens e 5 aspectos (fatores): aprender, antecipar, autorregular, harmonizar e apoiar. O alfa de Conbrach da escala é 0,764 . Conclusões: a escala de competência para o cuidado-5A retoma as abordagens da teoria da autogestão individual e familiar, avaliam de forma abrangente as habilidades requeridas nas pessoas com câncer para facilitar a transição em direção a autogestão do cuidado no lar.

Palavras chave: Neoplasias; Psicometria; Enfermagem Domiciliar; Autogestão 


\section{Introducción}

La incidencia de cáncer en el mundo está aumentando en todas las edades, se estima un aumento a 21.4 millones de casos para el 2020'. Sin embargo, mejores tratamientos han incrementado las tasas de supervivencia en el mundo, lo que desencadena sobrevivientes de cáncer viviendo con efectos a largo plazo derivados de las terapéuticas oncológicas. Al igual que en otras enfermedades crónicas, los efectos agudos y crónicos de los efectos del cáncer y su relación con los costos estarán generando dificultades para el sostenimiento de los actuales sistemas de salud ${ }^{2}$. Un mayor compromiso del paciente en el manejo de su condición de salud y autogestión especifica de sus comportamientos para reducir los efectos del tratamiento, son elementos clave para optimizar la atención en salud y mejorar la calidad de vida33.

Deotraparte, los pacientescon cáncerenfrentan periodos prolongados de toxicidadaltratamiento y síntomas relacionados por el tratamiento multimodal al que se ven sometidos, que puede conducir a complicaciones potencialmente mortales y a largo plazo a invalidez, particularmente si no se detectan tempranamente ni se manejan de forma efectiva. La modificación de estilos de vida y el manejo de los regímenes médicos en curso, y los comportamientos de prevención para reducir los efectos tardíos son una parte esencial del autocontrol efectivo de la enfermedad ${ }^{4}$.

Es de señalar que son los pacientes y sus familias, quienes deben asumir la responsabilidad de adoptar los comportamientos necesarios y desarrollar las competencias para manejar los síntomas del tratamiento, las consecuencias físicas y psicosociales, los cambios en el estilo de vida inherentes a su condición, para promover la supervivencia, recuperar la salud y el bienestar ${ }^{5}$.

Ser competente para el cuidado, es una expresión de las capacidades que debe desarrollar el paciente a través de un proceso continuo que involucra, el conocimiento de la situación, el entrenamiento en habilidades, el afianzamiento de la confianza y planeación necesarias para lograr un cuidado continuo y seguro, en las diferentes etapas de la condición.

El rol del equipo de salud, evoluciona como facilitador de este proceso de apoyo al paciente para que sea competente en el cuidado, a través de la ayuda en la implementación y mantenimiento de conductas para manejar la enfermedad ${ }^{6}$, adherirse a los regímenes terapéuticos y adaptarse a los cambios en los roles de vida, planes y actividades, así como manejar las consecuencias emocionales, y hacer frente a la incertidumbre y angustia que es común en el cáncer ${ }^{7}$.

Carrillo et al., ${ }^{8}$ desarrollaron el constructo de competencia para el cuidado en el hogar en personas con enfermedad crónica y sus cuidadores familiares, la definen como la capacidad, habilidad y preparación que tiene la persona con enfermedad crónica y/o el cuidador familiar para ejercer la ejercer la labor de cuidar en el hogar. Comprende 6 categorías que se ordenan bajo el acróstico CUIDAR : Conocimiento, Unicidad (condiciones personales), Instrumental, Disfrutar (bienestar), Anticipación, y Relación social

Ser competente para el cuidado, es una expresión de las capacidades que debe desarrollar el paciente a través de un proceso continuo que involucra, el conocimiento de la situación, el entrenamiento en habilidades, el afianzamiento de la confianza y planeación necesarias para lograr un cuidado continuo y seguro, en las diferentes etapas de la condición.

El rol del equipo de salud, evoluciona como facilitador de este proceso de apoyo al paciente para que sea competente en el cuidado, a través de la ayuda en la implementación y mantenimiento de conductas para manejar la enfermedad ${ }^{6}$, adherirse a los regímenes terapéuticos y adaptarse a los cambios en los roles de vida, planes y actividades, así como manejar las consecuencias emocionales, y hacer frente a la incertidumbre y angustia que es común en el cáncer?. 
e interacción. Posteriormente, se desarrollaron los instrumentos que miden 6 dimensiones y cuyo constructo, consta de 60 ítems ${ }^{9}$ y se derivó una escala de competencia para el cuidado en el hogar de personas con enfermedad crónica, versión abreviada que consta de 20 ítems, que permite optimizar el tiempo requerido para su aplicación, manteniendo su validez y confiabilidad.

Esta escala ha sido utilizada como indicador de proceso para orientar planes de alta y programas de egreso de personas con enfermedades crónicas y cuidadores familiares, se consolida como un referente para la atención durante la transición hospital-hogar que orienta la toma de decisiones del equipo de salud frente a las dimensiones que se deben afianzar en el paciente para que sea competente en el cuidado ${ }^{10}$.

Si bien, el cáncer es considerada una enfermedad crónica, se identifican condiciones particulares de la experiencia que vive el paciente durante el tratamiento oncológico, junto con la connotación y estigma que rodea al diagnóstico cáncer, que suponen un abordaje propio y diferente de los factores asociados a la competencia para el cuidado en el hogar, frente a otras condiciones crónicas.

El presente estudio buscó validar la estructura factorial hipotética de la escala competencia para el cuidado en el hogar en adultos con diagnóstico de cáncer que se encuentran en tratamiento activo.

\section{Metodología}

Estudio transversal de validación de instrumentos desarrollado entre los años 2019-2020, y que contó con el aval del Comité de ética institucional, mantuvo todos los requisitos exigidos por la Norma 8430 de $1993^{11}$, por la cual se establecen las pautas científicas, técnicas y administrativas para la investigación en salud en Colombia.

Para determinar la validez de constructo y la confiabilidad se aplicó la versión abreviada del instrumento competencia para el cuidado en el hogar a personas con cáncer ${ }^{8}$, consta de 20 ítems que se miden a través de escala tipo Likert con puntajes de 0 a 3 ( 0 nunca y 3 siempre), seis dimensiones: Conocimiento (3 ítems), Unicidad (4 ítems), Instrumental (3 ítems) , Disfrutar (4 ítems), Anticipación ( 2 ítems) , y Relación social e interacción ( 2 ítems). El muestreo fue probabilístico y aleatorio. El cálculo de tamaño de la muestra requerido se obtuvo de acuerdo con el número de ítems del instrumento, considerando un mínimo de 240 participantes (12 por ítem) $)^{12}$.

Los criterios de inclusión de los participantes fueron ser personas con diagnóstico de cáncer, en tratamiento activo contra el cáncer: quimioterapia y/o radioterapia, mayores de 18 años, que sepan leer y escribir y que asistan a una institución prestadora de servicios de salud.

La captación de los pacientes se hizo en los servicios de quimioterapia y/o radioterapia de la de la institución, a los participantes se les explicó el objetivo del estudio y se les aplicó el consentimiento informado. Posteriormente fueron abordados en un espacio físico que cumpliera condiciones de privacidad y concentración dentro de la institución. El proceso de recolección de datos estuvo a cargo de 3 auxiliares de investigación entrenados, la duración promedio de la aplicación de la escala fue de 15 a 20 minutos.

La validez de constructo se realizó por medio de la técnica de análisis factorial confirmatorio utilizando el método de componentes principales y rotación varimax. Se acogió lo descrito 
por Nunally y Bernstein ${ }^{12}$ respecto a los criterios en la evaluación de los ítems del instrumento con presencia de una carga factorial (loading) igual o superior a 0.3 con comunalidad al menos de 0.1 y en primera instancia ausencia de efecto adverso sobre las medidas de confiabilidad calculadas mediante el índice de Cronbach- Mesbah ${ }^{13}$, y en segunda, ausencia de correlación fuerte (superior a 0.8) y significancia con los demás ítems.

Para determinar la confiabilidad por medio de su nivel de consistencia interna se empleó el alfa de Cronbach ${ }^{14}$.

\section{Resultados}

Caracterización sociodemográfica de los pacientes con cáncer en tratamiento:

La muestra incluyó 250 personas ( $72.4 \%$ mujeres) entre 19 y 75 años (Media de edad $=50.34$, $\mathrm{SD}=12.831$ ), en su mayoría en los rangos de 36 a 59 años, de religión católica. Con respecto a la distribución por estrato socioeconómico se observó que la mayoría pertenece a estratos dos y tres. La mayoría de participantes reportó bachillerato como el nivel educativo completado (36.8\%). Los diagnósticos oncológicos más frecuentes fueron el cáncer de mama (38.8\%), linfomas $(11,2 \%)$ cáncer de colon $(9,3 \%)$, cáncer de ovario $(7,2 \%)$, y cáncer gástrico $(4,4 \%)$. El 78\% de los participantes no reporta metástasis. (Tabla 1)

\section{Tabla 1. Caracteristicas sociodemográficas de los participantes.}

\begin{tabular}{lll}
\hline Variable & & $\%$ \\
\hline Sexo & Masculino & 27,6 \\
Grupo de edad & Femenino & 72,4 \\
& 18 a 35 años & 14,0 \\
Religión & 36 a 59 años & 62,8 \\
& 60 a 74 años & 22,4 \\
& Mayor de 75 años & 0,8 \\
Escolaridad & Católico & 78,8 \\
& Cristiano & 14,4 \\
& Testigo de Jehová & 1,2 \\
Ninguna & 4,8 \\
Estrato socioeconómico & 28,4 \\
& Primaria & 36,8 \\
& Bachillerato & 14,8 \\
& Técnico & 14,0 \\
& Pregrado & 2,8 \\
& Especialización & 2,4 \\
Miagnóstico & 0,4 \\
& Maestría & 15,2 \\
& Estrato 2 & 42,4 \\
& Estrato 3 & 33,6 \\
& Estrato 4 & 7,6 \\
& Estrato 5 & 0,4 \\
& Cáncer de mama & 38.8 \\
& Cáncer de colon & 9,3 \\
& Cáncer de ovario & 7,2 \\
& Linfoma de Hodking & 6,8 \\
& Linfoma Hodking & 4,4 \\
& Cáncer deestómago & 4,4 \\
& Otros & 29,1 \\
& Sí & 16,0 \\
& No & 78,0 \\
\hline
\end{tabular}


Con respecto a la Validez de constructo y en concordancia con el método descrito se identificó el cumplimiento de tres condiciones necesarias para realizar el análisis factorial confirmatorio: se obtuvo una muestra de personas con cáncer mayor a 100 ( $\mathrm{N}=250$ participantes); un índice de medida de adecuación de la muestra (KMO), de 0,778, lo que indica que el análisis factorial es útil, y la prueba de esfericidad de Barlett de 0.00 (si Sig< 0.05 se puede aplicar el análisis factorial).

El análisis arrojó como resultado, seis factores con vector propio (eighenvalues) mayores a 1.00, y que en total lograron explicar el $60 \%$ de la varianza de las respuestas de los participantes.

A excepción de un ítem, en todas las preguntas del instrumento se observaron cargas factoriales superiores a 0.4. El quinto ítem "Supero fácilmente sentimientos de culpa o de rabia" presentó cargas factoriales pobres, indicando que no corresponde a ninguno de los factores arrojados por el análisis. De otra parte, aunque los ítems 8 "Actúo siguiendo las recomendaciones prescritas por el equipo de salud" y 10 "Uso adecuadamente los medicamentos formulados" presentaron cargas factoriales de 0.683 y 0.769 respectivamente, tuvieron una baja confiabilidad al ser analizados (0.294). Razón por la que se decide eliminarlos y volver a correr el análisis factorial confirmatorio con 5 factores.

El resultado comprende una estructura factorial compuesta por las cinco dimensiones. Esta estructura factorial logró explicar el $61.4 \%$ de la varianza en las respuestas de los participantes.

Frente a los componentes resultantes, el primero de ellos, denominado "aprender" está compuesto por 3 ítems referentes a la comprensión que tienen los participantes frente a la enfermedad, los cuidados y seguimiento a adelantar.

El segundo factor, denominado "anticipar" incluye tres ítems referentes a prever riesgos y necesidades de la atención, y establecer un plan de vida acorde con la condición de salud.

El tercer factor "auto-regular" corresponde a cuatro ítems que describen aspectos que permiten a la persona adaptarse a su condición de salud e incluyen la satisfacción personal, la confianza y acomodarse a las rutinas.

El cuarto factor "armonizar" refleja tres ítems que evidencian la organización de apoyos y recursos disponibles para las tareas del cuidado.

El factor cinco "apoyar" conformado por cuatro ítems, se refiere al establecimiento de redes de apoyo efectivas y comunicación con otros para favorecer la labor de cuidado. Tablas 2 y 3. 
Tabla 2. Matriz de componentes rotados. Competencia para el cuidado en el hogar adultos con cáncer.

\begin{tabular}{|c|c|c|c|c|c|}
\hline \multirow[b]{2}{*}{ Ítem } & \multicolumn{5}{|c|}{ Componentes } \\
\hline & Apoyar & $\begin{array}{l}\text { Auto } \\
\text { regular }\end{array}$ & Armonizar & Aprender & Anticipar \\
\hline $\begin{array}{l}\text { 20. Fortalezco vínculos de afecto con las personas } \\
\text { quienes apoyan mi cuidado }\end{array}$ & 0,905 & & & & \\
\hline $\begin{array}{l}\text { 19. Busco comunicarme efectivamente con las } \\
\text { personas quienes apoyan mi cuidado }\end{array}$ & 0,831 & & & & \\
\hline $\begin{array}{l}\text { 18. Mantengo tranquilidad emocional con las } \\
\text { personas que apoyan mi cuidado }\end{array}$ & 0,79 & & & & \\
\hline $\begin{array}{l}\text { 17. Logro respaldo de otras personas para apoyar } \\
\text { mi cuidado }\end{array}$ & 0,592 & & & & \\
\hline $\begin{array}{l}\text { 13. Siento que mi situación de salud me permite } \\
\text { desarrollar mi plan de vida }\end{array}$ & & 0,819 & & & \\
\hline $\begin{array}{l}\text { 14. El cuidado de mi salud no me impide desarrollar } \\
\text { otros asuntos o actividades personales }\end{array}$ & & 0,737 & & & \\
\hline $\begin{array}{l}\text { 12. Siento tener la energía para responder a las } \\
\text { responsabilidades del cuidado }\end{array}$ & & 0,687 & & & \\
\hline 11. Estoy satisfecho con mi estado de salud actual & & 0,682 & & & \\
\hline $\begin{array}{l}\text { 9. Organizo el apoyo instrumental disponible para } \\
\text { mi cuidado (Transporte, equipos, insumos, otros) }\end{array}$ & & & 0,795 & & \\
\hline 16. Organizo los recursos necesarios para mi cuidado & & & 0,692 & & \\
\hline 6. Soy capaz de simplificar las tareas del cuidado & & & 0,577 & & \\
\hline $\begin{array}{l}\text { 2. Conozco como realizar los procedimientos } \\
\text { requeridos para cuidarme }\end{array}$ & & & & 0,796 & \\
\hline $\begin{array}{l}\text { 3. Conozco los signos de alarma en mi condición } \\
\text { de salud }\end{array}$ & & & & 0,725 & \\
\hline $\begin{array}{l}\text { 1. Sé cómo hacer seguimiento de mis condiciones } \\
\text { de salud }\end{array}$ & & & & 0,649 & \\
\hline 7. Establezco mi propio plan de vida & & & & & 0,747 \\
\hline $\begin{array}{l}\text { 15. Preveo manejo de riesgos y de necesidades de } \\
\text { mi cuidado }\end{array}$ & & & 0,408 & & 0,642 \\
\hline $\begin{array}{l}\text { 4. Tengo confianza en mi capacidad para asumir } \\
\text { mi cuidado en casa }\end{array}$ & & & & & 0,493 \\
\hline
\end{tabular}

Nota: Método de extracción: análisis de componentes principales.

Método de rotación: Varimax con normalización Kaiser. 
Tabla 3. Propuesta estructura hipotética competencia para el cuidado en el hogar adultos con cáncer.

\begin{tabular}{|c|c|}
\hline Dimensión & Items \\
\hline \multirow[t]{3}{*}{ Aprender } & 1. Sé cómo hacer seguimiento de mis condiciones de salud \\
\hline & 2. Conozco como realizar los procedimientos requeridos para cuidarme \\
\hline & 3. Conozco los signos de alarma en mi condición de salud \\
\hline \multirow[t]{3}{*}{ Anticipar } & 4. Tengo confianza en mi capacidad para asumir mi cuidado en casa \\
\hline & 7. Establezco mi propio plan de vida \\
\hline & 15.Preveo manejo de riesgos y de necesidades de mi cuidado \\
\hline \multirow[t]{4}{*}{ Auto -regular } & 11.Estoy satisfecho con mi estado de salud actual \\
\hline & 12.Siento tener la energía para responder a las responsabilidades del cuidado \\
\hline & 13.Siento que mi situación de salud me permite desarrollar mi plan de vida \\
\hline & $\begin{array}{l}\text { 14.El cuidado de mi salud no me impide desarrollar otros asuntos o actividades } \\
\text { personales }\end{array}$ \\
\hline \multirow[t]{3}{*}{ Armonizar } & 6. Soy capaz de simplificar las tareas del cuidado \\
\hline & $\begin{array}{l}\text { 9. Organizo el apoyo instrumental disponible para mi cuidado (Transporte, equipos, } \\
\text { insumos, otros) }\end{array}$ \\
\hline & 16. Organizo los recursos necesarios para mi cuidado \\
\hline \multirow[t]{3}{*}{ Apoyar } & 17.Logro respaldo de otras personas para apoyar mi cuidado \\
\hline & 18. Mantengo tranquilidad emocional con las personas que apoyan mi cuidado \\
\hline & $\begin{array}{l}\text { 19. Busco comunicarme efectivamente con las personas quienes apoyan mi cuidado } \\
\text { 20. Fortalezco vínculos de afecto con las personas quienes apoyan mi cuidado }\end{array}$ \\
\hline
\end{tabular}

Nota: Los items 5, 8 y 10 de la escala original son eliminados en esta nueva estructura

La estructura hipotética propuesta en este estudio coincide con tres factores de la escala original CUIDAR para personas con enfermedad crónica desarrollada por Carrillo et al en $2016^{15}$, la de conocimiento, la de disfrutar y la de relación. Sin embargo, se observan discrepancias en ítems que se ubican en factores que derivaron en nuevas denominaciones de las categorías de la competencia para el cuidado en el hogar en adultos con cáncer. Tabla 4.

Tabla 4. Competencia para el cuidado en el hogar- estructura propuesta por Carrillo et al (2016) versus versión para adultos con cáncer Carrillo y Vargas (2020)

Competencia para el cuidado en el hogar

(Estructura hipotética) para personas con enfermedad crónica propuesta por Carrillo

et al. (2016)

Conocimiento: Ítems 1,2,3

Unicidad: Ítems 4,5,6,7

Disfrutar: Ítems 11,12,13,14

Anticipación: Ítems 15,16

Relación: Ítems 17,18,19,20
Competencia para el cuidado en el hogar para adultos con cáncer. (2020)

Análisis de confiabilidad

La confiabilidad por consistencia interna medida con el alfa de Cronbach fue de, 0.764 para el instrumento en su totalidad (17 ítems), con valores entre 0.588 y 0.780 en cada una de las dimensiones. Tabla 5 
Tabla 5. Confiabilidad de la Escala de competencia para el cuidado en el hogar en personas con cáncer

\begin{tabular}{ll}
\hline Competencia para el cuidado adultos con cáncer-dimensiones & Alfa de Cronbach \\
\hline Aprender & 0.609 \\
Armonizar & 0.657 \\
Auto-regular & 0.748 \\
Anticipar & 0.588 \\
Apoyar & 0.780 \\
Competencia para el cuidado & 0.764 \\
\hline
\end{tabular}

\section{Discusión}

El análisis factorial de este estudio muestra una solución de 5 sub-escalas de la competencia de cuidado en el hogar: aprender, armonizar, auto-regular, anticipar y apoyar. Los resultados discrepan de la estructura propuesta en la versión original del instrumento. Posibles explicaciones para esta estructura se derivan del abordaje hecho en adultos con cáncer que se encuentran en tratamiento activo contra el cáncer.

Esta nueva estructura aporta en la re-significación de los factores a explorar en las competencias que requieren las personas con cáncer, a la vez que permite orientar al paciente en la auto-evaluación de los "5 Aspectos" fundamentales para garantizar la autogestión de su condición oncológica. Recoge lo planteado por Ryan y Sawin ${ }^{16}$ en la teoría de automanejo individual y familiar, frente al proceso de autogestión, descrito como el uso de habilidades de autorregulación para gestionar afecciones crónicas o factores de riesgo. Estos procesos incluyen actividades como fijación de objetivos ${ }^{17}$, pensamiento reflexivo, toma de decisiones ${ }^{18}$, planificar y participar en comportamientos específicos, auto-evaluación y manejo de los aspectos físicos, emocionales y respuestas cognitivas asociadas con los cambios de comportamientos en salud. El individuo, entonces debe tomar el control y ser responsable del manejo de su condición en el desempeño de comportamientos aprendidos. Implica, entonces, conocimientos, creencias, capacidades de autorregulación y facilitación social ${ }^{19}$.

De esta manera, la intervención del equipo de salud trasciende los métodos de brindar información a facilitar el desarrollo de habilidades de autogestión desde las competencias para el cuidado.

El factor aprender, retoma los elementos de comprensión sobre la enfermedad y el tratamiento, sin embargo, se da la connotación como habilidad y acción del paciente, quien debe adquirir este conocimiento, a través de la información proporcionada por el equipo de salud y desde el reconocimiento de su experiencia.

En la sub-escala anticipar, se plantea la capacidad del paciente para prever riesgos y establecer su propio plan de vida, frente a su condición de salud. 
El factor armonizar, se orienta hacia el ajuste que debe realizar el paciente con cáncer en las tareas relacionadas con su condición de salud y la organización de apoyos y recursos necesarios para su cuidado. Incluye, además, habilidades instrumentales que debe desarrollar el paciente en cuanto a la administración de sus medicamentos, y las recomendaciones prescritas por el equipo de salud en las actividades de la vida diaria.

En la sub-escala autorregulación se exploran las capacidades del paciente para asumir su condición oncológica, resaltando la importancia de fortalecer autoconfianza, satisfacción, desarrollar actividades personales dentro de su plan de vida, y contar con la energía suficiente para responder por su cuidado.

El factor apoyar, retoma los elementos conceptuales del componente de relación e interacción de la escala original, en cuanto a las capacidades de comunicación, detección y uso de redes de apoyo primarias y secundarias.

Deacuerdocon estos hallazgos, la escala original es una herramienta útil para evaluar las competencias para el cuidado que requiere una persona en la transición hospital-hogar. Sin embargo, se recomiendan cambios en la estructura y conformación de las dimensiones, para personas con cáncer.

Se requiere continuar el proceso de validación de esta nueva estructura, en tal sentido, un segundo paso implica investigar la relevancia clínica de la solución de las 5 sub-escalas evaluando la capacidad de éstas para discriminar entre pacientes que reciben una intervención sobre la competencia para el cuidado en el hogar. Se requiere, además, explorar si la propuesta de las 5 A (Aprender, armonizar, auto-regular, anticipar, apoyar) es de mejor comprensión para los pacientes con cáncer, resultando en un efecto positivo en sus competencias para el cuidado, auto-confianza y apoyo con el equipo de salud.

\section{Conclusiones}

La escala competencia para el cuidado-5A retoma los planteamientos de la teoría de automanejo individual y familiar, evalúa de forma integral las habilidades requeridas en personas con cáncer para facilitar la transición hacia la autogestión del cuidado en el hogar. Es una escala que ofrece una nueva estructura de 5 factores (Aprender, armonizar, auto-regular, anticipar y apoyar) que aportan en la re-significación de los elementos fundamentales para garantizar la autogestión del paciente que cursa con una condición oncológica.

Conflicto de intereses: Ninguno

Financiación: Convocatoria nacional para el apoyo a proyectos de investigación y creación artística de la Universidad Nacional de Colombia 2017-2018. Universidad Nacional de Colombia. código de proyecto: 41763 


\section{Referencias}

1. The International Agency for Research on Cancer (IARC). New Global Cáncer Data: Globocan 2018. Disponible en:

https://www.uicc.org/news/new-global-cancer-data-globocan-2018

2. Kitts J, Beaton B, Cook C, Doiron C, Kendel D, McLeod L, et al,. Health Council of Canada Self-management support for Canadians with chronic health conditions: a focus for primary health care Toronto: Health Council of Canada, 2012. Disponible en: https://www.selfmanagementbc.ca/uploads/HCC_SelfManagementReport_FA.pdf

3. Henry D, Viswanathan H, Elkin E, Traina S, Wade S, Cella D. Symptoms and treatment burden associated with cancer treatment: results from a cross-sectional national survey in the US. Support Care Cancer. 2008;16(7):791-801 https://doi.org/10.1007/s00520-007-0380-2

4. Howell D, Harth T, Brown J, Bennett C, Boyko S. Self-management education interventions for patients with cancer: a systematic review. Support Care Cancer. 2017; 25(4): 1323-1355 https://doi.org/10.1007/s00520-016-3500-z

5. Phillips J, Currow D. Cancer as a chronic disease. Collegian. 2010;17(2):47-50 https://doi.org/10.1016/j.colegn.2010.04.007

6. Foster C, Fenlon D. Recovery and self-management support following primary cancer treatment. Br J Cancer. 2011; 105:S21-SS8 DOI:10.1038/bjc.2011.419

7. Howell D, Bezjak A, Sidani S, Dudgeon D, Husain A, Fernandez G et al. Self-management interventions for breathlessness in adult cancer patients. Cochrane Database Syst Rev. 2017 Aug; 2017(8): CD009623. doi: 10.1002/14651858.CD009623.pub2

8. Sánchez B, Carrillo G, Barrera L. El plan de transición y egreso hospitalario y su efecto en el cuidado de la salud: una revisión integrada. Rev. U.D.C.A. Act. Div.Cient. 2014; 17(1): 13-23

9. Carrillo G, Sánchez B, Arias E. Validation of an instrument to assess the homecare competency of the family caregiver of a person with chronic disease. Invest. Educ. Enferm. 2015; 33(3): 449-455

10.Melo B, Vargas Y, Carrillo G, Alarcón D. Efecto del programa Plan de egreso en la díada persona con enfermedad crónica y sus cuidadores familiares. Enferm Clín. 2018; 28 (1): 36-43. https:// doi.org/10.1016/j.enfcli.2017.09.006

11. Ministerio de Salud y Protección Social. Resolución n. 008430 de 1993, por la cual se establecen las normas científicas, técnicas y administrativas para la investigación en salud (04/10/1993). Disponible en: https://www.minsalud.gov.co/sites/rid/Lists/BibliotecaDigital/ RIDE/DE/DIJ/RESOLUCION-8430-DE-1993.PDF

12.Nunnally J, Bernestein H. Teoría Psicométrica. Tercera Edición. México: Mc Graw-Hill. 1995.

13.Mesbah M. Statistical quality of life., Method an Aplications of Statistics in the Life and Health Sciences. Balakrishnan (Editor).New Jersey: Wiley. 2010.

14.Campo A, Oviedo H. Propiedades psicométricas de una escala: la consistencia interna. Rev Salud Pública. 2008;10(5):831-9

15.Carrillo G, Sánchez B, Vargas E. Desarrollo y pruebas psicométricas del Instrumento "cuidar" - versión corta para medir la competencia de cuidado en el hogar. Salud UIS. 2016; 48(2): 222-231

16.Ryan P, Sawin K. The Individual and Family Self-Management Theory: Background and perspectives on context, process, and outcomes. Nurs Outlook. 2009;57(4):217-225 DOI: https://doi.org/10.1016/j.outlook.2008.10.004

17.Lorig K, Holman H. Self-management and education: History, definition, outcomes, and mechanisms. Ann Behav Med. 2003;26(1):1-7.

https://doi.org/10.1207/S15324796ABM2601_01 
18.Barlow J, Sturt J, Hearnshaw H. Self-management interventions for people with chronic conditions in primary care: Examples from arthritis, asthma and diabetes. Health Educ J. 2002;61:365-78 https://doi.org/10.1177/001789690206100408

19.Bodenheimer T, Wagner $\mathrm{E}$, Grumbach K. Improving primary care for patients with chronic illness. JAMA. 2002; 288 (14):1775-9. doi:10.1001/jama.288.14.1775 